\title{
Memory search: When does semantic analysis occur?*
}

\author{
MARILYN C. SMITH and SHARON M. ABEL† \\ Scarborough College, University of Toronto, West Hill, Ontario, Canada
}

\begin{abstract}
This study was addressed to the determination of the stage at which semantic analysis occurs during a STM recognition task. A list of exemplars (memory set), drawn from 13 different categories, was presented at a rate of $11 / 2 \mathrm{scc} / \mathrm{item}$, followed by a category name probe. Ss then indicated whether any of the exemplars in the memory set belonged to that category. List length was varied from 5 to 7 , with each memory set containing items from either two or three categories. It was found that RT was independent of list length, but did increase by $60 \mathrm{msec}$ as the number of categories represented in the list increased from two to three. From this, it was concluded that activation of a superordinate category representation occurred upon presentation of each memory-set item, and that the memory scan was done on these superordinate categories.
\end{abstract}

During the past few years. extensive investigation has been done of the processing stages involved in short-term memory (STM). Much of this work has used a paradigm initiated by Sternberg (1966). In this procedure, Ss are presented with a list of words (memory set) and are then asked whether a given probe item, presented following the list, was or was not a member of the memory set. It is generally found that the time required to categorize a probe as belonging or not belonging to the memory set increases linearly with the size of the set. From this finding. Sternberg concluded that a serial scan was done of the items in memory, with the intercept representing the time to encode the stimulus and execute the response and the slope representing the time required for individual memory comparisons.

While semantic analysis of the words is typically not required for the performance of the task, several recent studies suggest that such analysis does occur. De Rosa and Morin (1970) found that when the memory set consisted of a set of consecutive digits, the time required to determine that the probe digit was not a member of this set decreased as the difference between the arithmetic value of the presented stimulus and the highest (or lowest) digit in the memory set increased. Also, Lively and Sanford (1972) found that if the memory set consisted of items from a given category (digits or letters), then negative RTs were faster to noncategory probes than to probes from the same category (flatter slope). Both of these studies tend to indicate fairly extensive analysis of the memory-set items. Juola and Atkinson (1971) presented Ss with a list of category names as the memory set, and an

*This research was supported by National Research Council Grant APA-191 to Marilyn Smith. The authors are indebted to Drs. Bert Forrin and $\mathrm{Kim}$ Kirsner for their helpful comments on an earlier draft of this paper.

tPresent address: Department of Otolaryngology. Sunnybrook Hospital. University of Toronto. 2075 Bayview Avenue. Toronto 12. Ontario. Canada. exemplar of one of these categories as the probe. Ss were required to indicate whether the category to which the exemplar belonged was a member of the positive set. They found an increase both in slope and intercept when the list consisted of category names as compared with the condition where the memory set consisted of a list of exemplars. This would suggest that in a situation which requires the Ss to analyze for meaning, there is still a linear scan of memory, albeit at a slower rate.

Given that semantic analysis is occurring in such a STM recognition task, this paper is addressed to the problem of determining the stage at which this analysis occurs. Ss were presented with an auditory list of words (memory set) whose length was varied from five to seven. These words were selected from 13 categories. A probe was then presented which consisted of a category name. $S$ was required to make a positive response if that category had been represented in the memory set and a negative response if it had not. Thus, the situation required that each of the memory-set items be analyzed for meaning.

Such semantic analysis could occur at different stages. Some of these possibilities are listed below.

(1) Categorization during the memory comparison stage. One strategy which Ss might employ is to retain the original memory set in an uncategorized form. Then. upon presentation of the category name probe. this memory set is scanned. with the semantic analysis occurring during the memory comparison stage. Thus. for example, if the probe word is "profession," $\mathrm{S}$ would go through the memory set asking, "Is a dog a profession?" "Is a dentist a profession?" etc. If the semantic analysis occurs at the memory comparison stage, one prediction which must be made is that RT should increase with list length. Failure to find this increase would mean that the analysis occurred elsewhere.

(2) Categorization of items during momori-set presentation. A second possibility is that each of the memory-set items is cutegorized upon presentation. This 
would be in accordance with the suggestion of Collins and Quillian (1970), that upon presentation of a word, activation occurs of the superordinate category name for that word. When the probe is then presented, the scan is done not of the original items, but rather of these higher order representations. This could be done in two ways. One alternative is that there is a single internal representation of a given superordinate category. Thus, whenever an exemplar of a given category is presented, the same higher order internal representation is activated. Alternatively, it may be that Ss store each stimulus word in a complex which contains both the category information and the original stimulus representation, e.g., dog-animal, dentist-profession, etc. Such a complex has been suggested by Lively and Sanford (1972). These two alternatives can be differentiated by varying the number of instances of a given category. If the same superordinate representation is activated by every instance of that category, then memory scan should be independent of list length and dependent only upon the number of categories represented in the list. On the other hand, if the Lively and Sanford "complex" representation occurs, list length should be an important variable and we would expect the number of instances to be unimportant.

(3) Exemplar generation upon presentation of the probe. A different strategy which Ss could adopt is to generate the list of exemplars in a category when the category name probe is presented. Morin et al (1970) have presented data which suggest that this occurs when the category is a small one (e.g., directions-north, south, east, west). Ss would then compare the list of exemplars with each of the memory-set items. Again, one would expect increases in RT as a function of memory-set size, and further, RT should be greater for probe words representing large categories than for those representing smaller ones.

This study is an attempt to determine which of these models best describes the stage at which semantic analysis occurs in a STM recognition task.

\section{METHOD}

\section{Subjects}

Six undergraduates at Scarborough College were paid to serve as $S$ s in the experiment. No $S$ reported a hearing disorder that might result in speech distortion and none had previously participated in a memory experiment.

\section{Apparatus}

The Ss were tested individually in a sound-attenuated cubicle. They listened monaurally (right ear) through a headset driven by magnetic tape on a Sony (TC-355) four-track tape recorder. Tapes consisted of prerecorded word lists. RTs to probe words in each list were measured to the nearest millisecond using a Hunter Klockounter (Model 120A, Series D). During the recording stage, prior to speaking the probe word, E depressed a manual switch which activated a voice-operated relay key (Uhr Model F422). At the onset of the probe word, this key marked the tape in a second channel. An oscilloscope showed that across probe words, the time between probe onset and tape mark ranged between 20 and $40 \mathrm{msec}$. During the playback stage, a sensing device in the relay key triggered the timer at these marks. However, because of the distance between recording and playback heads on the recorder used, a constant lag of $267 \mathrm{msec}$ was introduced between the start of the timer and the presentation of the probe word. Consequently, this value was subtracted from all RT measures. A pair of microswitches manually operated by $S$ were used to stop the clock and to indicate a choice of alternative responses.

\section{Procedure}

Ss participated individually in seven experimental sessions, each lasting $1 \frac{1}{2} \mathrm{~h}$. During the first session, $\mathrm{S}$ was asked to study 13 lists, each consisting of instances of a particular category. The categories employed were animals, birds, boys' names, cars, cities, clothing, fish, flowers, foods, girls' names, household items, insects, and professions. The categories were compiled by selecting common items from each category, of ten consulting books which specifically dealt with one of the categories, e.g., a text on insects or on birds. Following the study period, $\mathrm{S}$ was tested on his ability to correctly categorize a random set of 25 instances which $E$ read aloud to him. Study and test sets were continued until $S$ had successfully categorized all the items in three successive sets.

During each of Sessions 2-7, S was presented with three blocks of 72 trials. List length within each block was held constant at five, six, or seven items. Half the lists were positive and half were negative. Order of presentation of the three blocks was balanced across sessions and Ss.

Within each list, choice of exemplars was restricted to either two or three different categories. It was necessary to limit the number of categories to two in this way in order to orthogonally vary number of categories, set size, and number of instances. For example, with Set Size 5 , in order to have three instances of a given category, we could not use more than three categories. Similarly, we could not have only one category, or the number of instances would equal set size. The 10 larger categories ( 50 to 100 instances) were represented equally of ten across lists within each block. Three categories with fewer than 50 instances (i.e., birds, fish, insects) were used together as often as one of the larger categories. Presentation of the list was followed by a category name probe word.

For the 36 positive lists within each block, the category probed was represented by one, two, or three specific instances. The remaining items in the list were distributed among the unprobed categories (whether one or two). Within a block of trials, for each combination of number of categories and number of positive instances $(2 \times 3)$, there were six lists. Within these six lists, the positive instances (i.e., instances representing the probed category) were either clustered (three lists) or unclustered (three lists). In clustered lists, positive instances appeared adjacent to one another at the beginning, middle, or end of the list. In the unclustered lists, positive instances varied in degree of proximity to each other.

The three degrees of proximity for unclustered positive instances depended on both number of positive instances and set size. Models for these lists are given in Table 1. For lists with only one positive instance, the instance appeared at the beginning, middle, or end position in each of two lists. Where lists contained items from two unprobed categories, these categories were represented equally of ten where possible. Within each positive list, negative instances of similar type were grouped.

The 36 negative lists within a block matched positive lists in all respects except that a category word not represented in the list appeared as the probe. For each list, Ss were required to decide whether an instance of the category probe had appeared in the list. Half the Ss signified "yes" with a microswitch held in the right hand, half with a microswitch held in the left hand. 
Decision RT was recorded. All S, heard the same tapes. The tapes used for the three blocks of trials varied across sessions.

Items in each list were presented at the rate of one word every $11 / 2$ sec. The probe followed the final item by 3 sec. A warning signal (pencil tap) was presented $13 \mathrm{sec}$ prior to the first item and $11 / 2 \mathrm{sec}$ following the final item in the list. Trials were seprated by approximately 6 sec.

\section{RESULTS}

A preliminary analysis of results indicated that across Ss the mean RT for combinations of set size and response type (positive and negative) decreased about $260 \mathrm{msec}$ from Session 1 to Session 2. From Session 2 through Session 6. RT remained fairly constant at $333 \mathrm{msec}$ for positive responses and at about $463 \mathrm{msec}$ for negative responses. In view of this practice effect, the data for Session 1 were discarded. For the remaining sessions. only those RTs associated with correct responses have been analyzed.

Figure 1 shows the RT to positive and negative probes for each of the three set sizes. Each data point is the mean RT for the six Ss to approximately 180 lists each. Since the data for all Ss were essentially the same, their results have been pooled. It is apparent from the graph that RT to both negative and positive lists is constant for the three levels of list length. The mean RT for negative probes is about $130 \mathrm{msec}$ greater than the mean RT for positive probes. This absence of a set-size effect was consistent across all days and did not change as a function of practice.

Figure 2 shows the RT to positive and negative probes for lists with words taken from two and three categories.

Table 1

Models for Clustering Positive Instances in Lists With Two Categories

\begin{tabular}{|c|c|c|c|c|c|c|}
\hline \multicolumn{2}{|r|}{ Two Instances } & & & \multicolumn{3}{|c|}{ Three Instances } \\
\hline & & & Set Size 5 & & & \\
\hline+ & $x$ & + & & + & + & + \\
\hline $\mathrm{x}$ & + & $\mathrm{x}$ & & $x$ & + & + \\
\hline$x$ & $x$ & $\mathrm{x}$ & & + & $\mathrm{x}$ & $\mathrm{x}$ \\
\hline+ & $\mathrm{x}$ & $\mathrm{x}$ & Set Size 6 & + & $\mathrm{x}$ & + \\
\hline $\mathrm{x}$ & + & $\mathrm{x}$ & & $x$ & + & $\mathrm{x}$ \\
\hline $\mathrm{x}$ & $\mathrm{x}$ & + & & + & $x$ & $\mathrm{x}$ \\
\hline $\mathrm{X}$ & $x$ & $\mathrm{x}$ & & $x$ & + & + \\
\hline $\mathrm{x}$ & + & + & & $x$ & $\mathrm{x}$ & $\mathrm{x}$ \\
\hline+ & $\mathrm{x}$ & $x$ & & + & + & + \\
\hline & & & Set Size 7 & & & \\
\hline+ & $x$ & $\mathrm{x}$ & & + & $\mathrm{x}$ & $\mathrm{x}$ \\
\hline$x$ & + & $\mathrm{x}$ & & $x$ & + & $\mathrm{x}$ \\
\hline $\mathrm{x}$ & $\mathrm{x}$ & + & & $x$ & $x$ & + \\
\hline $\mathrm{x}$ & $x$ & $\mathrm{x}$ & & + & $\mathrm{x}$ & $\mathrm{x}$ \\
\hline$x$ & $x$ & + & & $x$ & + & + \\
\hline $\mathrm{x}$ & + & $x$ & & $x$ & $x$ & $x$ \\
\hline+ & $x$ & $x$ & & + & + & + \\
\hline
\end{tabular}

Note-" +" refers to instances of the probed category.

*In lists with three categories. half the negative items $(x)$ represented each of the mo unprobed categories. Given an odd number of xs. one unprobed categon was represented by one item more than the other.

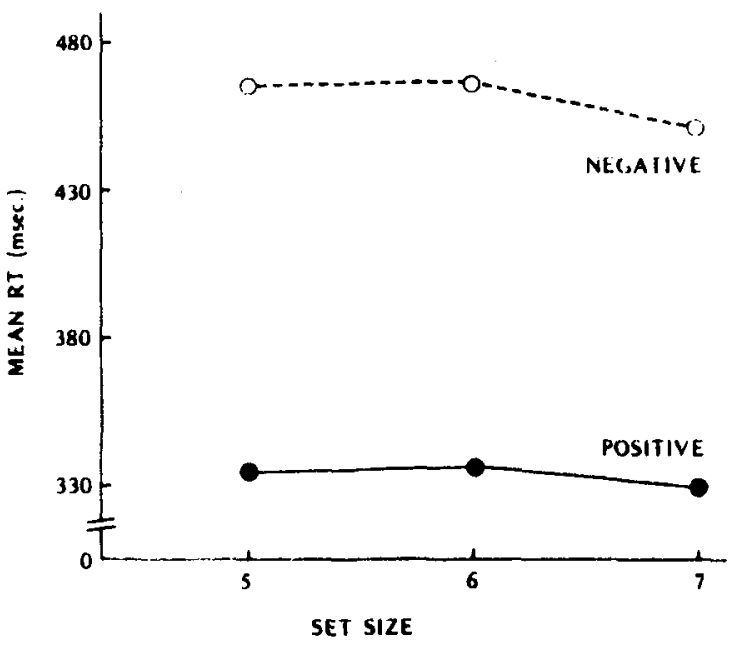

Fig. 1. Mean RT as a function of set size for positive and negative responses.

Each data point is the mean RT for the six Ss to approximately 270 lists each. Across response types, RT increases about $60 \mathrm{msec}$ as number of categories increases from two to three.

A repeated measures analysis of variance was performed on the mean RT for combinations of levels of set size $(5,6,7)$, number of categories $(2,3)$, number of instances $(1,2,3)$, and response type (positive, negative). Each mean represented in the design was based on RTs to approximately 30 lists ( 6 lists $\times 5$ sessions). The results of this test indicated that the following effects were significant beyond the .01 level of confidence: response type, $F(1,5)=200.88$; number of categories, $F(1,5)=56.11$; Response Type by Number of Instances, $F(2,10)=16.74$; and Set Size by Number of Categories by Number of Instances, $F(4,20)=4.76$. The significant interactions when plotted did not show meaningful trends. The effects of set size, $F(2,10)=.23$. number of instances, $F(2,10)=5.96$, and Number of Categories by Response Type, $F(1,5)=1.51$, were not significant at the .01 level. While the effects of number of instances was significant at the .05 level of confidence, this effect is difficult to interpret. since plotting RT as a function of number of instances yields a curvilinear relationship, with fastest RTs to one and three instances and largest $\mathrm{RT}$ to two instances.

A second repeated measures analysis of variance showed that the difference in mean RT for clustered and unclustered positive instances given two or three categories in positive lists was not significant at the .01 level. $F(1.5)=4.76$

The mean percent errors were calculated for the 36 positive and negative lists within each block for Sessions 2-6. The average percent errors across sessions was 5.6 for positive lists. with a range of 4.2 to 8.6 across Ss. and was 5.3 for negative lists. with a range of 2.2 to 16.1 across Ss. These arrors did not appear to be related to any particular treatment combination. 


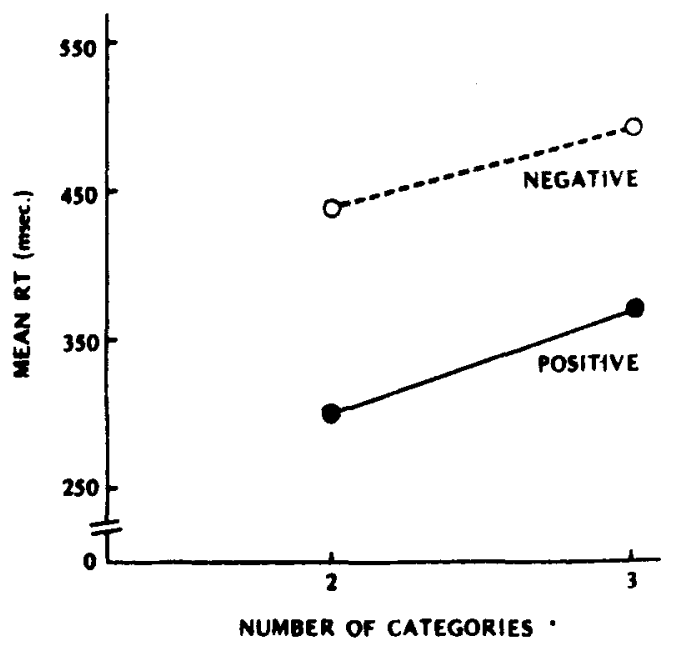

Fig. 2. Mean RT as a function of number of categories for positive and negative responses.

\section{DISCUSSION}

The present study was designed to determine the stage at which semantic encoding occurs in a STM recognition task. The finding that RT was unaffected by the length of the memory set rules out several possible strategies which Ss might have employed. First, it indicates that categorization of the words does not occur during the memory comparison stage. Second, the result makes unlikely the possiblity suggested by Lively and Sanford that the memory representation of an item is a complex consisting of both the item and its category name. Third, it implies that duplicate category names are not generated for instances from the same superordinate class. Finally, the finding that RT does not increase with increases in list length eliminates the possibility that upon presentation of the probe, Ss are generating the list of exemplars and comparing this list with the items in the memory set.

Rather, the joint results of no effect on RT of memory-set size combined with an increase in RT with number of categories represented in the list supports the hypothesis that a superordinate category name is generated for each item during presentation of the list, and that it is these category names which are then employed during the memory search.

One question of interest in this study was how the RT to a positive category name probe would vary as a function of the number of exemplars from that category. Assuming that on each presentation of a category exemplar the same superordinate category name is generated, then one might expect an increase in the trace strength of that category representation with the number of times activation occurred, and hence a decrease in RT when that category name was probed. Such a result would be in accordance with the finding of Baddeley and Ecob (1970) that when a memory set contained repeated items, RT to a probe which replicated a repeated item was faster than RT to the probe for a nonrepeated item. However, in the present study, the number of instances of the category probe had no effect on RT. We also found that having the positive instances clustered rather than distributed throughout the list did not affect RT. This result would indicate that repeated generation of the superordinate category representation does not result in greater trace strength for that representation.

In order to determine whether a scanning of category names would occur in a situation where $S$ s are required to maintain the original items in memory, an additional $S$ was run, who was required, on a random one-third of the trials, to recall the original list after making his response. Again, RT showed no effect of set size, but did increase with the number of categories. Recall of the memory set was almost perfect. It would be of some interest to determine whether similar results would be found in a slightly different situation, one in which the test stimulus on any trial could be either a category name or simply a word from the memory set, with a cue for response criterion presented just prior to test stimulus onset. One possibility is that the typical increasing functions with increasing set size would be found for physical match probes and the results of the present study would be duplicated for category name probes. Such results would be consistent with a partitioned memory store, with each partition accessed separately. ${ }^{1}$

Finally, it should be noted that the RTs found in this experiment tend to be lower than those typically found in memory search experiments. This may be due to the greater practice of the Ss in this study, since RTs for the first day alone closely resemble the values typically found.

\section{REFERENCES}

Baddeley, A. D., \& Ecob, J. R. Reaction time and short-term memory: A trace strength alternative to the high-speed exhaustive scanning hypothesis. CHIP Report 13, 1970.

Collins, A. M., \& Quillian, M. R. Does category size affect categorization time? Journal of Verbal Learning \& Verbal Behavior, $1970,9,432-438$.

De Rosa, D. V., \& Morin, R. E. Recognition reaction time for digits in consecutive and nonconsecutive memorized sets. Journal of Experimental Psychology, 1970, 83, 472-479.

Juola, J. F., \& Atkinson, R. C. Memory scanning for words versus categories. Journal of Verbal Learning \& Verbal Behavior, 1971, 10, 522-527.

Lively, B. L., \& Sanford, B. J. The use of category information in a memory-search task. Journal of Experimental Psychology, 1972, 93, 370-385.

Morin, R. E., Hoving, K. L., \& Konick, D. S. Are these two stimuli from the same set? Response times of children and adults for stimuli from familiar and arbitrary sets. Journal of Experimental Child Psychology, 1970, 10, 308-318.

Sternberg, S. High-speed scanning in human memory. Science, $1966,153,652-654$. 


\section{NOTE}

1. We are indebted to the reviewer of this article for this suggestion.
(Rectived for publication August 4, 1972; revision received September 20, 1972.) 